The Agriculturists 14(1): 83-91(2016) ISSN 2304-7321 (Online), ISSN 1729-5211 (Print)

A Scientific Journal of Krishi Foundation

Indexed Journal

Impact Factor: 0.402 (GIF, 2014)

\title{
Effect of Storage Condition on Seed Quality of Some Dhaincha (Sesbania spp.) Accessions
}

\author{
M. Towhidul Karim ${ }^{1}$, Fazlul Haque 2 , A. K. M. Golam Sarwar ${ }^{3}$ and M. Ayub Ali ${ }^{4}$ \\ ${ }^{1}$ Departments of Seed Science and Technology, ${ }^{3}$ Crop Botany and ${ }^{4}$ Plant Pathology, Bangladesh \\ Agricultural University, Mymensingh; ${ }^{2}$ Seed and Agro Enterprise, BRAC, Dhaka, Bangladesh
}

*Corresponding author and Email: drsarwar@bau.edu.bd

Received: 12 April $2016 \quad$ Accepted: 12 June 2016

\begin{abstract}
An experiment was conducted at the Seed Pathology Laboratory, Department of Plant Pathology, Bangladesh Agricultural University, Mymensingh, to understand the effect of storage conditions on seed quality of different accessions of dhaincha (Sesbania spp.). Seeds of twenty accessions were collected and stored in a refrigerator $\left(4^{\circ} \mathrm{C}\right)$ and laboratory at normal room temperature $\left(25-30^{\circ} \mathrm{C}\right)$. Sprouting (\%), germination (\%), vigour index and the incidence of seed-borne pathogens were recorded following ISTA procedures. Germination and emergence were higher when seeds were stored in the refrigerator than those in room temperature. The vigour index was also high when the seeds were stored in a refrigerator. Fungi belonging to genera Aspergillus, Fusarium, Rhizopus, and Penicillium were associated with seeds of the different accessions of dhaincha. Seed borne infection of Fusarium sp. was highest $(14 \%)$ in G-13 when seeds were stored at room temperature. It was evident from the results that the quality of the seeds of dhaincha accessions was retained for a long time when they were stored at low (around $4^{0} \mathrm{C}$ ) temperature.
\end{abstract}

Keywords: Dhaincha, storage condition, seed quality, seed health

\section{Introduction}

Dhaincha (Sesbania spp.), a member of the family Fabaceae, is an ideal green manure crop in Bangladesh (Sarwar et al., 2015). The genus Sesbania, consists 60 species encompassing annuals, perennials, herbs, shrubs and trees, is distributed in the tropical and subtropical climates (Veasey et al., 1999). The perennial species of Sesbania establish easily and can grow under stress conditions with minimum care. They have many attributes that make them attractive as multipurpose plants and potentially useful species in agricultural production systems (Sarwar et al., 2015). Three species, viz. Sesbania sesban (L.) Merr., S. bispinosa (Jacq.)
Wight and S. cannabina (Retz.) Poir are commonly known as "Dhaincha" in Bangladesh (Ahmed et al., 2009).

Seed deterioration is inexorable and non-uniform (McDonald, 2004). Many factors contribute to seed deterioration including genetic composition, seed moisture content, mechanical and insect damage, pathogen attack, seed maturity, and relative humidity and temperature of the storage environment. However, relative humidity and temperature are considered as the most important factors that regulate the seed quality (McDonald, 2004; Basavegowda et al., 2013). Relative humidity directly influences seed moisture. At low temperature $\left(5-7^{\circ} \mathrm{C}\right)$, pests incidence are 
low, as a result, seed viability and quality remain satisfactory during storage time (Croft et al., 2012). The storage container also affects the quality of seeds at storage (Monira et al., 2012). Similar observations on the superiority of impervious container (polythene lined gunny bag) for maintenance of soybean seeds quality was observed by Sharma et al. (1998). Alam et al. (2010) had similar findings in case of bean seeds. Dwivedi and Shukla (1990) also narrated similar observations in chickpea. Croft et al. (2012) noted seed germination as a function of the duration of storage, air temperature and seed moisture content at storage. Therefore, the study was conducted to determine the effect of storage condition on the health, germination, and emergence of seeds of different dhaincha accessions.

\section{Materials and Methods}

Seeds of twenty dhaincha accessions were collected from different parts of Bangladesh in November 2014 (collection information would be available upon request to corresponding author). Seed samples were sun dried, cleaned and divided into two halves. One-half of the seed samples of each accession were stored at room temperature and the other half was stored in a refrigerator at the Plant Systematics Laboratory, Department of Crop Botany, Bangladesh Agricultural University, Mymensingh. In both cases, seeds were in air tight plastic bottles. The temperature inside the refrigerator was $4^{\circ} \mathrm{C}$ and that in the room temperature was $25-30^{\circ} \mathrm{C}$. The seeds were stored for 6 months. At the end of the storage period, samples were collected and the seeds were subjected to germination and health test at the Seed Pathology Laboratory, Department of Plant Pathology, Bangladesh Agricultural University, Mymensingh.

\subsection{Germination test}

Germination test was done in the sterile sand medium in plastic containers. Four hundred seeds of each accession were tested. One hundred seeds were placed in a container and considered as one replication. Thus, there were four replications. The number of normal and abnormal seedlings, dead and non-germinated seeds were counted after 10 days of seed sowing. Percentage of germination was calculated. Root and shoot length $(\mathrm{cm})$ of 20 seedlings were measured. Seedling vigour was determined following Baki and Anderson (1972):

Vigour Index $=($ Root length + Shoot length $) \times$ Germination (\%)

\subsection{Seed health test}

Seed health tests were done by Dry Inspection and Blotter Incubation method following ISTA (2001) procedure. In the dry inspection, the seeds of each accession were categorized into healthy, small and shriveled seeds on the basis of visual diagnosis with the aid of hand lens. Blotter incubation test was done on Whatman No. 1 blotter paper contained in plastic petridishes. Three layer of water soaked blotter papers were placed on each petridishes and 25 seeds/dish were placed. The seeds were incubated in the incubation chamber. After five days, number of seeds with mycelia colonies was counted. The genera of fungi were identified under the compound microscope at 40x, with the help of manuals of seed borne fungi.

\subsection{Data Analysis}

Data were analyzed statistically following the analysis of variance (ANOVA) technique using MS-Stat computer package and means were separated by Duncan's new multiple range test (DMRT) at 5\% level of significance.

\section{Results and Discussion}

Storage condition particularly temperature had a profound influence on seed germination and emergence of dhaincha seeds of all accessions (Table 1). The highest number of normal seedlings $(76 \%)$ was recorded in G-13, while the lowest percentage $(21.75 \%)$ was in G-20 when the seeds were stored in the refrigerator. The highest percentage of normal seedlings $(69.50 \%)$ was recorded in G-5 and the lowest $(21.50 \%)$ was in G-20 when the seeds were stored at room temperature. Seed emergence was $90 \%$ in G-11 
and $30.75 \%$ in G-20 when the seeds were stored in the refrigerator. Emergence was $81.50 \%$ in G05 , while only $26.50 \%$ in G-20 when the seeds were stored at room temperature. Variations in emergence and seedling attributes among the accessions of dhaincha might be due to the genetic make-up of the respective accessions. There was a significant difference between the percentage of emergence and normal seedlings within the accessions stored in a refrigerated condition (Table 1). There was no significant difference in emergence percentage between G11 and G-13, but the difference was significant in the percentage of normal seedlings. That kind of variation was not observed in seeds stored at room temperature. The highest emergence $(\%)$ of G-20 seeds was maintained up to two months from the date of withdrawal from the refrigerated condition as reported by Karim (2015). The results of this investigation regarding the effect of different storage conditions were in conformity with that of the earlier researchers (Dwivedi and Shukla, 1990; Alam et al., 2010; Basavegowda et al., 2010).

There was a significant difference in vigour index among the dhaincha accessions and under different storage conditions (Table 2). Vigour index was higher when seeds were stored in a refrigerator and was lower when the seeds stored at room temperature. The highest vigour index (2002) was recorded in G-11, while the lowest (494.8) in G-20 when the seeds were stored in the refrigerator (Table 2). Vigour index was 1448 in G-05 and 391.2 in G-20 when the seeds were stored at the room temperature. In the refrigerator, physiological activities of the seeds were supposed to reduce due to low temperature, which caused an enhanced seed longevity. The probable reason for the slow rate of reduction in germination and vigour in cold storage condition could be due to reduced rate of respiration and metabolic changes occurring in seeds (Das et al. 1998).

The highest percentage of healthy seeds $(83 \%)$ was recorded in G-06 and G-08, and the lowest $(50 \%)$ was in G-20, when the seeds were stored in refrigerator. These two accessions, G-06 and G-08 also produced relatively low percentage of shriveled and small seeds in the refrigerated condition (Table 3). The highest percentage of healthy $(81.75 \%)$ seeds was observed in G-11, while the poorest $(47.50 \%)$ was in G-20 when the seeds were stored at room temperature. Accession G-20 produced the highest percentage of shriveled and small seeds in both refrigerated and room temperature conditions (Table 3).

Four fungal genera viz. Aspergillus, Fusarium, Penicillium, and Rhizopus were identified in the stored seeds of different accessions of dhaincha (Table 4). Among them, Fusarium and Rhizopus emerged as the most prevalent and damaging genera for stored dhaincha seeds. Legume seeds might be susceptible to these two fungal genera. Khalequzzaman (2004) reported from a study that $19.99 \%$ and $19.65 \%$ French bean seeds were infected by Fusarium spp. and Rhizopus sp., respectively. Aspergillus and Penicillium are mostly responsible for the deterioration of stored seed of rice, wheat, pulses, oil seeds and different vegetable crops as reported by Copeland (1976).

In all the cases, fungal incidence was higher when seeds were stored at room temperature. When the seeds were preserved in refrigerated condition, the incidence of Fusarium sp. and Rhizopus sp. ranged from $4 \%$ to $11 \%$ followed by $A$. flavus $1 \%$ to $4 \%$, Penicillium sp. $0 \%$ to $3 \%$ and A. niger $0 \%$ to $2 \%$ (Table 4 ). On the contrary, when seeds were stored at room temperature the incidence of Fusarium sp. ranged from $7 \%$ to $14 \%$ followed by Rhizopus sp. $6 \%$ to $12 \%$, A. flavus $2 \%$ to $5 \%$, Penicillium sp. $0 \%$ to $4 \%$ and $A$. niger $0 \%$ to $3 \%$. Christensen (1967) observed that freshly harvested duram wheat seeds stored at $25^{\circ} \mathrm{C}$ with 13.4-13.6\% moisture for 493 days were slowly invaded by $A$. halophilicus and $A$. restrictus, though germination was not affected. However, when seeds were stored with a moisture content of $14.2-14.4 \%$, population of storage fungi increased and germination percentage was decreased noticeably. 
Table 1. Effect of storage condition on seed germination of different accessions of dhaincha

\begin{tabular}{|c|c|c|c|c|c|c|}
\hline $\begin{array}{l}\text { Storage } \\
\text { Condition }\end{array}$ & $\begin{array}{l}\text { Accession } \\
\text { number }\end{array}$ & $\begin{array}{l}\text { Emergence } \\
(\%)\end{array}$ & $\begin{array}{l}\text { Normal } \\
\text { seedlings } \\
(\%)\end{array}$ & $\begin{array}{l}\text { Abnormal } \\
\text { seedlings } \\
(\%)\end{array}$ & $\begin{array}{l}\text { Dead } \\
\text { seeds } \\
(\%) \\
\end{array}$ & $\begin{array}{l}\text { Un- } \\
\text { germinated } \\
\text { seeds }(\%)\end{array}$ \\
\hline \multirow{20}{*}{ 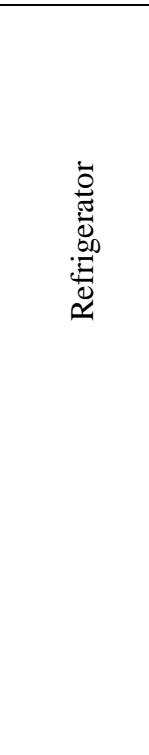 } & G-01 & $73.50 \mathrm{hi}$ & $60.50 \mathrm{ef}$ & $13.00 \mathrm{gh}$ & $12.15 \mathrm{~h}-\mathrm{j}$ & $15.00 \mathrm{jk}$ \\
\hline & G-02 & $75.05 \mathrm{gh}$ & $58.00 \mathrm{f}-\mathrm{h}$ & $17.05 \mathrm{c}$ & $8.00 \mathrm{o}$ & $17.00 \mathrm{i}$ \\
\hline & G-03 & $60.00 \mathrm{pq}$ & $49.001-n$ & $11.00 \mathrm{ij}$ & $15.00 \mathrm{de}$ & $25.00 \mathrm{e}$ \\
\hline & G-04 & $77.13 \mathrm{~g}$ & $60.00 \mathrm{ef}$ & $17.13 \mathrm{c}$ & $10.11 \mathrm{~m}$ & $13.00 \mathrm{~lm}$ \\
\hline & G-05 & $81.13 \mathrm{f}$ & $71.13 \mathrm{~b}$ & $10.00 \mathrm{jk}$ & 8.00 no & $12.00 \mathrm{mn}$ \\
\hline & G-06 & 81.75 ef & $62.25 \mathrm{de}$ & $19.50 \mathrm{~b}$ & $5.00 \mathrm{qr}$ & $14.00 \mathrm{kl}$ \\
\hline & G-07 & 81.75 ef & $71.75 \mathrm{~b}$ & $10.00 \mathrm{jk}$ & $9.10 \mathrm{mn}$ & 11.00 no \\
\hline & G-08 & $83.75 \mathrm{~d}-\mathrm{f}$ & $63.50 \mathrm{~d}$ & $20.25 \mathrm{~b}$ & $6.00 \mathrm{pq}$ & 11.00 no \\
\hline & G-09 & $84.71 \mathrm{c}-\mathrm{e}$ & $69.50 \mathrm{~b}$ & $15.21 \mathrm{~d}$ & 7.00 op & $9.00 \mathrm{pq}$ \\
\hline & G-10 & $86.50 \mathrm{~b}-\mathrm{d}$ & $66.25 \mathrm{c}$ & $20.25 \mathrm{~b}$ & $5.00 \mathrm{qr}$ & $9.00 \mathrm{pq}$ \\
\hline & G-11 & $90.00 \mathrm{a}$ & $70.50 \mathrm{~b}$ & $19.50 \mathrm{~b}$ & $4.00 \mathrm{rs}$ & $6.00 \mathrm{r}$ \\
\hline & G-12 & $66.281-n$ & $54.25 \mathrm{ij}$ & $12.02 \mathrm{hi}$ & $14.00 \mathrm{ef}$ & $20.00 \mathrm{~h}$ \\
\hline & G-13 & $89.13 \mathrm{ab}$ & $76.00 \mathrm{a}$ & $13.13 \mathrm{gh}$ & $3.00 \mathrm{~s}$ & $9.00 \mathrm{pq}$ \\
\hline & G-14 & $64.88 \mathrm{~m}-\mathrm{o}$ & $50.88 \mathrm{k}-\mathrm{m}$ & $14.00 \mathrm{e}-\mathrm{g}$ & $15.00 \mathrm{de}$ & $23.63 \mathrm{e}-\mathrm{g}$ \\
\hline & G-15 & $87.50 \mathrm{a}-\mathrm{c}$ & $75.50 \mathrm{a}$ & $12.00 \mathrm{hi}$ & $5.00 \mathrm{qr}$ & $8.00 \mathrm{q}$ \\
\hline & G-16 & $64.75 \mathrm{~m}-\mathrm{o}$ & $51.25 \mathrm{kl}$ & $13.50 \mathrm{fg}$ & $13.00 \mathrm{f}-\mathrm{h}$ & $23.75 \mathrm{ef}$ \\
\hline & G-17 & 63.38 no & $48.00 \mathrm{mn}$ & $15.38 \mathrm{~d}$ & $14.00 \mathrm{ef}$ & $23.50 \mathrm{e}-\mathrm{g}$ \\
\hline & G-18 & $73.31 \mathrm{hi}$ & $62.25 \mathrm{de}$ & $11.06 \mathrm{ij}$ & $11.00 \mathrm{kl}$ & $16.00 \mathrm{ij}$ \\
\hline & G-19 & $64.25 \mathrm{~m}-\mathrm{o}$ & $53.25 \mathrm{jk}$ & $11.00 \mathrm{ij}$ & 14.00 ef & $22.00 \mathrm{~g}$ \\
\hline & G-20 & $30.75 \mathrm{t}$ & $21.75 \mathrm{q}$ & $9.00 \mathrm{kl}$ & $29.13 \mathrm{a}$ & $41.25 \mathrm{~b}$ \\
\hline \multirow{20}{*}{ 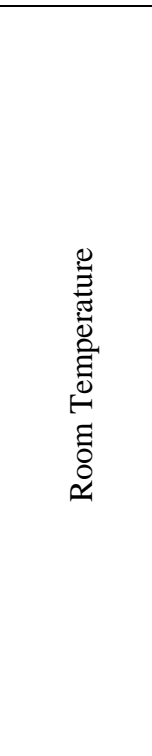 } & G-01 & 70.00 i-k & $59.00 \mathrm{fg}$ & $11.00 \mathrm{ij}$ & $12.21 \mathrm{~g}-\mathrm{i}$ & $18.81 \mathrm{~h}$ \\
\hline & G-02 & $65.50 \mathrm{~m}-\mathrm{o}$ & $44.00 \mathrm{o}$ & $21.50 \mathrm{a}$ & $15.25 \mathrm{~d}$ & $20.00 \mathrm{~h}$ \\
\hline & G-03 & $57.00 \mathrm{qr}$ & $47.00 \mathrm{n}$ & $10.00 \mathrm{jk}$ & $20.13 \mathrm{bc}$ & $23.00 \mathrm{fg}$ \\
\hline & G-04 & $56.40 \mathrm{r}$ & 42.00 op & $14.40 \mathrm{~d}-\mathrm{f}$ & $15.00 \mathrm{de}$ & $29.00 \mathrm{~d}$ \\
\hline & G-05 & 81.50 ef & $69.50 \mathrm{~b}$ & $12.00 \mathrm{hi}$ & $9.1 \mathrm{mn}$ & 10.00 op \\
\hline & G-06 & $69.00 \mathrm{j}-1$ & $49.001-n$ & $20.00 \mathrm{~b}$ & $11.36 \mathrm{i}-\mathrm{k}$ & $20.00 \mathrm{~h}$ \\
\hline & G-07 & $67.75 \mathrm{k}-\mathrm{m}$ & $50.001-n$ & $17.75 \mathrm{c}$ & $13.16 \mathrm{f}-\mathrm{h}$ & $19.38 \mathrm{~h}$ \\
\hline & G-08 & $72.25 \mathrm{~h}-\mathrm{j}$ & $54.25 \mathrm{ij}$ & $18.00 \mathrm{c}$ & $12.00 \mathrm{~h}-\mathrm{k}$ & $16.00 \mathrm{ij}$ \\
\hline & G-09 & $73.00 \mathrm{hi}$ & $56.00 \mathrm{~h}-\mathrm{j}$ & $17.00 \mathrm{c}$ & $13.30 \mathrm{fg}$ & $14.00 \mathrm{kl}$ \\
\hline & G-10 & $72.00 \mathrm{~h}-\mathrm{j}$ & $57.00 \mathrm{~g}-\mathrm{i}$ & $15.00 \mathrm{de}$ & $15.00 \mathrm{de}$ & $13.00 \mathrm{~lm}$ \\
\hline & G-11 & $70.13 \mathrm{i}-\mathrm{k}$ & $50.001-n$ & $20.13 \mathrm{~b}$ & $14.00 \mathrm{ef}$ & $16.00 \mathrm{ij}$ \\
\hline & G-12 & $49.00 \mathrm{~s}$ & $40.00 \mathrm{p}$ & $9.00 \mathrm{kl}$ & $20.42 \mathrm{bc}$ & $31.00 \mathrm{c}$ \\
\hline & G-13 & $77.00 \mathrm{~g}$ & $55.00 \mathrm{ij}$ & $22.00 \mathrm{a}$ & $11.00 \mathrm{j}-1$ & $12.21 \mathrm{mn}$ \\
\hline & G-14 & $47.50 \mathrm{~s}$ & $39.50 \mathrm{p}$ & 8.001 & $21.00 \mathrm{~b}$ & $32.00 \mathrm{c}$ \\
\hline & G-15 & $75.00 \mathrm{gh}$ & $55.00 \mathrm{~h}-\mathrm{j}$ & $20.00 \mathrm{~b}$ & $12.13 \mathrm{~h}-\mathrm{j}$ & $13.18 \mathrm{~lm}$ \\
\hline & G-16 & $50.50 \mathrm{~s}$ & $41.50 \mathrm{op}$ & $9.00 \mathrm{kl}$ & $21.00 \mathrm{~b}$ & $28.00 \mathrm{~d}$ \\
\hline & G-17 & $57.25 \mathrm{qr}$ & $51.25 \mathrm{kl}$ & $6.00 \mathrm{mn}$ & $14.00 \mathrm{ef}$ & $29.36 \mathrm{~d}$ \\
\hline & G-18 & $65.00 \mathrm{~m}-\mathrm{o}$ & $50.00 \mathrm{n}$ & $15.00 \mathrm{de}$ & $11.00 \mathrm{j}-1$ & $24.00 \mathrm{ef}$ \\
\hline & G-19 & $62.13 \mathrm{op}$ & $56.00 \mathrm{~h}-\mathrm{j}$ & $6.13 \mathrm{~m}$ & $14.00 \mathrm{ef}$ & $24.00 \mathrm{ef}$ \\
\hline & G-20 & $26.50 \mathrm{u}$ & $21.50 \mathrm{q}$ & $5.00 \mathrm{n}$ & $19.38 \mathrm{c}$ & $60.00 \mathrm{a}$ \\
\hline $\mathrm{LSD}_{0.05}$ & & 2.66 & 3.12 & 1.05 & 1.03 & 1.51 \\
\hline $\mathrm{CV}(\%)$ & & 3.48 & 3.24 & 5.35 & 5.84 & 5.52 \\
\hline
\end{tabular}


Table 2. Effect of storage condition on vigor index of different accessions of dhaincha

\begin{tabular}{|c|c|c|c|c|c|}
\hline $\begin{array}{l}\text { Storage } \\
\text { condition }\end{array}$ & $\begin{array}{l}\text { Accession } \\
\text { number }\end{array}$ & $\begin{array}{l}\text { Germination } \\
(\%)\end{array}$ & $\begin{array}{l}\text { Average root } \\
\text { length }(\mathrm{cm})\end{array}$ & $\begin{array}{l}\text { Average shoot } \\
\text { length }(\mathrm{cm})\end{array}$ & Vigour index \\
\hline \multirow{20}{*}{ 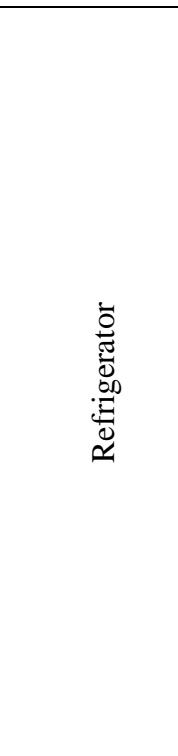 } & G-01 & 60.50 ef & $4.93 \mathrm{mn}$ & $13.19 \mathrm{bc}$ & $1323.00 \mathrm{f}$ \\
\hline & G-02 & $58.00 \mathrm{f}-\mathrm{h}$ & $5.65 \mathrm{~h}-\mathrm{j}$ & $11.90 \mathrm{e}-\mathrm{j}$ & $1316.00 \mathrm{f}$ \\
\hline & G-03 & $49.001-n$ & $4.27 \mathrm{pq}$ & $11.40 \mathrm{~h}-1$ & 939.80 o-q \\
\hline & G-04 & 60.00 ef & $3.90 \mathrm{rs}$ & $10.601-p$ & $1116.00 \mathrm{kl}$ \\
\hline & G-05 & $71.13 \mathrm{~b}$ & $5.86 \mathrm{hi}$ & $12.80 \mathrm{c}-\mathrm{e}$ & $1493.00 \mathrm{~cd}$ \\
\hline & G-06 & $62.25 \mathrm{de}$ & $4.25 \mathrm{pq}$ & $11.30 \mathrm{i}-\mathrm{m}$ & $1260.00 \mathrm{f}-\mathrm{h}$ \\
\hline & G-07 & $71.75 \mathrm{~b}$ & 4.80 no & $12.90 \mathrm{~cd}$ & $1416.00 \mathrm{e}$ \\
\hline & G-08 & $63.50 \mathrm{~d}$ & $5.95 \mathrm{f}-\mathrm{h}$ & $8.51 \mathrm{q}$ & $1200.00 \mathrm{~h}-\mathrm{j}$ \\
\hline & G-09 & $69.50 \mathrm{~b}$ & $4.92 \mathrm{mn}$ & $10.30 \mathrm{n}-\mathrm{p}$ & $1278.00 \mathrm{fg}$ \\
\hline & G-10 & $66.25 \mathrm{c}$ & $5.90 \mathrm{~g}-\mathrm{i}$ & $11.95 \mathrm{e}-\mathrm{i}$ & $1535.00 \mathrm{bc}$ \\
\hline & G-11 & $70.50 \mathrm{~b}$ & $7.54 \mathrm{c}$ & $14.70 \mathrm{a}$ & $2002.00 \mathrm{a}$ \\
\hline & G-12 & $54.25 \mathrm{ij}$ & $7.03 \mathrm{~d}$ & $10.92 \mathrm{k}-\mathrm{o}$ & $1185.00 \mathrm{i}-\mathrm{k}$ \\
\hline & G-13 & $76.00 \mathrm{a}$ & $5.021-n$ & $12.50 \mathrm{c}-\mathrm{g}$ & $1542.00 \mathrm{bc}$ \\
\hline & G-14 & $50.88 \mathrm{k}-\mathrm{m}$ & $8.60 \mathrm{a}$ & $12.72 \mathrm{c}-\mathrm{f}$ & $1322.00 \mathrm{f}$ \\
\hline & G-15 & $75.50 \mathrm{a}$ & $5.80 \mathrm{~h}-\mathrm{j}$ & $12.30 \mathrm{c}-\mathrm{h}$ & $1575.00 \mathrm{~b}$ \\
\hline & G-16 & $51.25 \mathrm{kl}$ & $6.50 \mathrm{e}$ & $13.90 \mathrm{~b}$ & $1306.00 \mathrm{f}$ \\
\hline & G-17 & $48.00 \mathrm{mn}$ & 6.24 ef & $11.70 \mathrm{~g}-\mathrm{k}$ & $1130.00 \mathrm{j}-1$ \\
\hline & G-18 & $62.25 \mathrm{de}$ & $4.00 \mathrm{q}$ & $8.45 \mathrm{q}$ & $912.50 \mathrm{qr}$ \\
\hline & G-19 & $53.25 \mathrm{jk}$ & $6.50 \mathrm{e}$ & $12.00 \mathrm{~d}-\mathrm{i}$ & $1184.00 \mathrm{i}-\mathrm{k}$ \\
\hline & G-20 & $21.75 \mathrm{q}$ & $4.00 \mathrm{q}-\mathrm{s}$ & $12.50 \mathrm{c}-\mathrm{g}$ & $494.80 \mathrm{t}$ \\
\hline \multirow{20}{*}{ 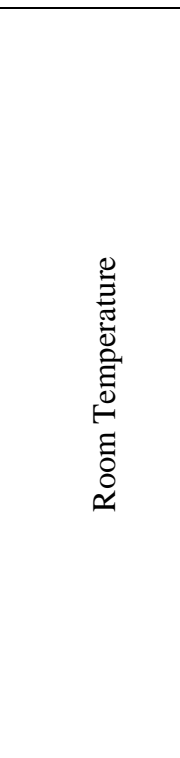 } & G-01 & $59.00 \mathrm{fg}$ & $4.50 \mathrm{p}$ & $13.05 \mathrm{c}$ & $1230.00 \mathrm{~g}-\mathrm{i}$ \\
\hline & G-02 & $44.00 \mathrm{o}$ & $5.60 \mathrm{ij}$ & $11.50 \mathrm{~h}-\mathrm{k}$ & 1111.001 \\
\hline & G-03 & $47.00 \mathrm{n}$ & $4.00 \mathrm{q}-\mathrm{s}$ & $11.01 \mathrm{j}-\mathrm{n}$ & $855.60 \mathrm{r}$ \\
\hline & G-04 & 42.00 op & $3.69 \mathrm{~s}$ & $9.77 \mathrm{p}$ & $755.40 \mathrm{~s}$ \\
\hline & G-05 & $69.50 \mathrm{~b}$ & $5.78 \mathrm{~h}-\mathrm{j}$ & $12.10 \mathrm{~d}-\mathrm{i}$ & $1448.00 \mathrm{de}$ \\
\hline & G-06 & $49.001-n$ & $4.10 \mathrm{qr}$ & $10.30 \mathrm{n}-\mathrm{p}$ & 993.60 n-p \\
\hline & G-07 & $50.001-n$ & $4.31 \mathrm{pq}$ & $11.90 \mathrm{e}-\mathrm{j}$ & $1102.00 \mathrm{~lm}$ \\
\hline & G-08 & $54.25 \mathrm{ij}$ & $5.25 \mathrm{kl}$ & $8.20 \mathrm{q}$ & $974.80 \mathrm{n}-\mathrm{q}$ \\
\hline & G-09 & $56.00 \mathrm{~h}-\mathrm{j}$ & $4.15 \mathrm{qr}$ & 10.13 n-p & $1042.00 \mathrm{mn}$ \\
\hline & G-10 & $57.00 \mathrm{~g}-\mathrm{i}$ & $5.50 \mathrm{jk}$ & $11.34 \mathrm{i}-\mathrm{m}$ & $1212.00 \mathrm{~g}-\mathrm{i}$ \\
\hline & G-11 & $50.001-n$ & $4.30 \mathrm{pq}$ & 10.10 op & 1008.00 no \\
\hline & G-12 & $40.00 \mathrm{p}$ & $6.90 \mathrm{~d}$ & $10.50 \mathrm{~m}-\mathrm{p}$ & $852.60 \mathrm{r}$ \\
\hline & G-13 & $55.00 \mathrm{ij}$ & 4.56 op & $11.90 \mathrm{e}-\mathrm{j}$ & $1267.00 \mathrm{f}-\mathrm{h}$ \\
\hline & G-14 & $39.50 \mathrm{p}$ & $8.10 \mathrm{~b}$ & $11.80 \mathrm{f}-\mathrm{k}$ & $930.40 \mathrm{pq}$ \\
\hline & G-15 & $55.00 \mathrm{~h}-\mathrm{j}$ & $5.20 \mathrm{~lm}$ & $12.10 \mathrm{~d}-\mathrm{i}$ & $1306.00 \mathrm{f}$ \\
\hline & G-16 & $41.50 \mathrm{op}$ & $6.20 \mathrm{e}-\mathrm{g}$ & $13.15 \mathrm{bc}$ & 986.80 n-p \\
\hline & G-17 & $51.25 \mathrm{kl}$ & $5.95 \mathrm{f}-\mathrm{h}$ & $11.56 \mathrm{~h}-\mathrm{k}$ & 1011.00 no \\
\hline & G-18 & $50.00 \mathrm{n}$ & $3.85 \mathrm{rs}$ & $8.23 \mathrm{q}$ & $785.20 \mathrm{~s}$ \\
\hline & G-19 & $56.00 \mathrm{~h}-\mathrm{j}$ & 6.25 ef & $11.90 \mathrm{e}-\mathrm{j}$ & $1125.00 \mathrm{kl}$ \\
\hline & $\mathrm{G}-20$ & $21.50 \mathrm{q}$ & $3.90 \mathrm{rs}$ & $11.70 \mathrm{~g}-\mathrm{k}$ & $391.20 \mathrm{u}$ \\
\hline $\mathrm{LSD}_{0.05}$ & & 2.66 & 0.276 & 0.766 & 64.58 \\
\hline $\mathrm{CV}(\%)$ & & 3.48 & 3.70 & 4.78 & 4.02 \\
\hline
\end{tabular}


Table 3. Seed health status of the seeds of 20 accessions of dhaincha (dry inspection)

\begin{tabular}{|c|c|c|c|c|}
\hline Storage Condition & $\begin{array}{c}\text { Accession } \\
\text { number }\end{array}$ & $\begin{array}{c}\text { Healthy Seeds } \\
(\%)\end{array}$ & $\begin{array}{c}\text { Shriveled Seeds } \\
(\%)\end{array}$ & $\begin{array}{c}\text { Small Seeds } \\
(\%)\end{array}$ \\
\hline \multirow{20}{*}{ 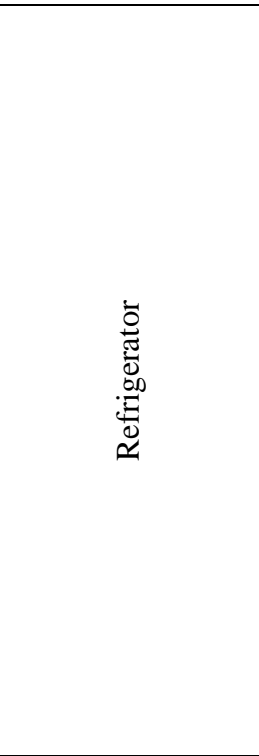 } & G-01 & $79.25 \mathrm{a}-\mathrm{g}$ & $13.75 \mathrm{np}$ & 7.02 h-k \\
\hline & G-02 & $72.50 \mathrm{k}-\mathrm{n}$ & $21.25 \mathrm{~d}$ & $6.25 \mathrm{kl}$ \\
\hline & G-03 & $78.75 \mathrm{a}-\mathrm{h}$ & $13.75 \mathrm{n}-\mathrm{p}$ & $7.50 \mathrm{hi}$ \\
\hline & G-04 & $74.50 \mathrm{~h}-\mathrm{m}$ & $16.75 \mathrm{jk}$ & $8.75 \mathrm{~g}$ \\
\hline & G-05 & 80.50 a-e & $13.25 \mathrm{o}-\mathrm{q}$ & $6.25 \mathrm{kl}$ \\
\hline & G-06 & $83.00 \mathrm{a}$ & $10.00 \mathrm{v}$ & $7.00 \mathrm{~h}-\mathrm{k}$ \\
\hline & G-07 & $78.75 \mathrm{a}-\mathrm{h}$ & $15.25 \mathrm{~lm}$ & 6.001 \\
\hline & G-08 & $83.00 \mathrm{a}$ & $12.25 \mathrm{q}-\mathrm{s}$ & $7.25 \mathrm{~h}-\mathrm{j}$ \\
\hline & G-09 & $79.50 \mathrm{a}-\mathrm{f}$ & $11.50 \mathrm{r}-\mathrm{u}$ & $9.00 \mathrm{~g}$ \\
\hline & G-10 & $81.25 \mathrm{a}-\mathrm{e}$ & $14.00 \mathrm{~m}-\mathrm{o}$ & $4.75 \mathrm{~m}$ \\
\hline & G-11 & $82.50 \mathrm{ab}$ & $12.50 \mathrm{p}-\mathrm{r}$ & $5.00 \mathrm{~m}$ \\
\hline & G-12 & $70.25 \mathrm{~m}-\mathrm{o}$ & $20.25 \mathrm{de}$ & $9.50 \mathrm{fg}$ \\
\hline & G-13 & $81.50 \mathrm{a}-\mathrm{d}$ & $11.00 \mathrm{~s}-\mathrm{V}$ & $7.50 \mathrm{hi}$ \\
\hline & G-14 & $73.75 \mathrm{j}-\mathrm{n}$ & $19.00 \mathrm{e}-\mathrm{g}$ & $7.25 \mathrm{~h}-\mathrm{j}$ \\
\hline & G-15 & 80.75 a-e & $10.50 \mathrm{uv}$ & $8.75 \mathrm{~g}$ \\
\hline & G-16 & $82.00 \mathrm{a}-\mathrm{c}$ & $10.75 \mathrm{t}-\mathrm{v}$ & $7.25 \mathrm{~h}-\mathrm{j}$ \\
\hline & G-17 & $70.25 \mathrm{~m}-\mathrm{o}$ & $12.50 \mathrm{p}-\mathrm{r}$ & $17.25 \mathrm{a}$ \\
\hline & G-18 & $73.50 \mathrm{j}-\mathrm{n}$ & $19.25 \mathrm{ef}$ & $7.25 \mathrm{~h}-\mathrm{j}$ \\
\hline & G-19 & $75.00 \mathrm{~g}-1$ & $18.50 \mathrm{f}-\mathrm{i}$ & $6.50 \mathrm{j}-\mathrm{l}$ \\
\hline & G-20 & $50.00 \mathrm{pq}$ & $37.50 \mathrm{c}$ & $12.50 \mathrm{c}$ \\
\hline \multirow{20}{*}{ 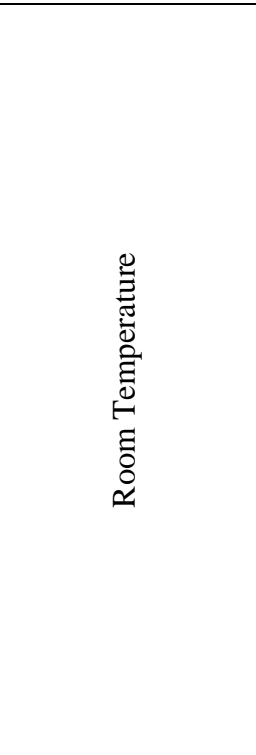 } & G-01 & $77.50 \mathrm{c}-\mathrm{j}$ & $15.75 \mathrm{kl}$ & $6.75 \mathrm{i}-1$ \\
\hline & G-02 & $52.25 \mathrm{p}$ & $39.00 \mathrm{~b}$ & $8.75 \mathrm{~g}$ \\
\hline & G-03 & $75.00 \mathrm{~g}-1$ & $12.50 \mathrm{p}-\mathrm{r}$ & $12.50 \mathrm{c}$ \\
\hline & G-04 & $70.00 \mathrm{no}$ & $12.50 \mathrm{p}-\mathrm{r}$ & $17.50 \mathrm{a}$ \\
\hline & G-05 & $75.50 \mathrm{f}-1$ & $17.00 \mathrm{jk}$ & $7.50 \mathrm{hi}$ \\
\hline & G-06 & $74.00 \mathrm{i}-\mathrm{n}$ & $17.25 \mathrm{ij}$ & 10.00 ef \\
\hline & G-07 & $77.25 \mathrm{~d}-\mathrm{j}$ & $14.00 \mathrm{~m}-\mathrm{o}$ & $8.75 \mathrm{~g}$ \\
\hline & G-08 & 80.00 a-e & $15.001-n$ & $5.00 \mathrm{~m}$ \\
\hline & G-09 & $74.50 \mathrm{~h}-\mathrm{m}$ & $17.75 \mathrm{~g}-\mathrm{j}$ & $7.75 \mathrm{~h}$ \\
\hline & G-10 & $79.00 \mathrm{a}-\mathrm{g}$ & $14.00 \mathrm{~m}-\mathrm{o}$ & $7.00 \mathrm{~h}-\mathrm{k}$ \\
\hline & G-11 & $81.75 \mathrm{a}-\mathrm{d}$ & $12.00 \mathrm{q}-\mathrm{t}$ & $6.25 \mathrm{kl}$ \\
\hline & G-12 & 70.00 no & $17.50 \mathrm{~h}-\mathrm{j}$ & $12.50 \mathrm{c}$ \\
\hline & G-13 & 80.00 a-e & $13.75 n-p$ & $6.25 \mathrm{kl}$ \\
\hline & G-14 & $70.50 \mathrm{~m}-\mathrm{o}$ & $15.75 \mathrm{kl}$ & $13.75 \mathrm{~b}$ \\
\hline & G-15 & $78.25 \mathrm{~b}-\mathrm{i}$ & $18.75 \mathrm{f}-\mathrm{h}$ & $3.00 \mathrm{n}$ \\
\hline & G-16 & $76.75 \mathrm{e}-\mathrm{k}$ & $15.001-n$ & $7.75 \mathrm{~h}$ \\
\hline & G-17 & $67.50 \mathrm{o}$ & $21.25 \mathrm{~d}$ & $11.50 \mathrm{~d}$ \\
\hline & G-18 & $70.00 \mathrm{~m}-\mathrm{o}$ & $21.25 \mathrm{~d}$ & $8.75 \mathrm{~g}$ \\
\hline & G-19 & $72.251-\mathrm{n}$ & $17.25 \mathrm{ij}$ & $10.50 \mathrm{e}$ \\
\hline & G-20 & $47.50 \mathrm{q}$ & $41.25 \mathrm{a}$ & $8.75 \mathrm{~g}$ \\
\hline $\mathrm{LSD}_{0.05}$ & & 3.74 & 1.21 & 0.748 \\
\hline $\mathrm{CV}(\%)$ & & 3.59 & 5.08 & 6.30 \\
\hline
\end{tabular}


Table 4. Effect of storage conditions on seedborne fungus infection of different accessions of dhaincha

\begin{tabular}{|c|c|c|c|c|c|c|}
\hline $\begin{array}{l}\text { Storage } \\
\text { condition }\end{array}$ & $\begin{array}{l}\text { Accession } \\
\text { number }\end{array}$ & $\begin{array}{l}\text { Aspergillus } \\
\text { flavus (\%) }\end{array}$ & $\begin{array}{l}\text { Aspergillus } \\
\text { niger }(\%)\end{array}$ & $\begin{array}{l}\text { Fusarium } \\
\text { sp. (\%) }\end{array}$ & $\begin{array}{l}\text { Penicillium } \\
\text { sp. }(\%)\end{array}$ & $\begin{array}{l}\text { Rhizopus } \\
\text { sp. (\%) }\end{array}$ \\
\hline \multirow{20}{*}{ 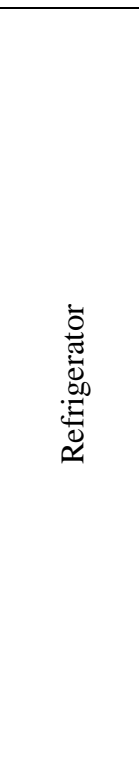 } & G-01 & $2.00 \mathrm{~d}$ & $0.00 \mathrm{~d}$ & $6.00 \mathrm{j}$ & $0.00 \mathrm{e}$ & $4.00 \mathrm{i}$ \\
\hline & G-02 & $1.00 \mathrm{e}$ & $2.00 \mathrm{~b}$ & $7.12 \mathrm{hi}$ & $2.00 \mathrm{c}$ & $7.00 \mathrm{f}$ \\
\hline & G-03 & $2.00 \mathrm{~d}$ & $0.00 \mathrm{~d}$ & $6.00 \mathrm{j}$ & $2.00 \mathrm{c}$ & $5.00 \mathrm{~h}$ \\
\hline & G-04 & $2.00 \mathrm{~d}$ & $2.00 \mathrm{~b}$ & $6.00 \mathrm{j}$ & $0.00 \mathrm{e}$ & $6.00 \mathrm{~g}$ \\
\hline & G-05 & $2.01 \mathrm{~d}$ & $1.00 \mathrm{c}$ & $7.00 \mathrm{i}$ & $1.00 \mathrm{~d}$ & $4.00 \mathrm{i}$ \\
\hline & G-06 & $2.00 \mathrm{~d}$ & $1.00 \mathrm{c}$ & $8.00 \mathrm{gh}$ & $0.00 \mathrm{e}$ & $7.00 \mathrm{f}$ \\
\hline & G-07 & $3.00 \mathrm{c}$ & $2.00 \mathrm{~b}$ & $5.00 \mathrm{k}$ & $2.00 \mathrm{c}$ & $5.00 \mathrm{~h}$ \\
\hline & G-08 & $2.00 \mathrm{~d}$ & $1.00 \mathrm{c}$ & $4.00 \mathrm{k}$ & $0.00 \mathrm{e}$ & $6.00 \mathrm{~g}$ \\
\hline & G-09 & $3.00 \mathrm{c}$ & $0.00 \mathrm{~d}$ & $7.00 \mathrm{i}$ & $2.00 \mathrm{c}$ & $8.00 \mathrm{e}$ \\
\hline & G-10 & $1.00 \mathrm{e}$ & $0.00 \mathrm{~d}$ & 9.00 ef & $2.00 \mathrm{c}$ & $9.00 \mathrm{~d}$ \\
\hline & G-11 & $1.00 \mathrm{e}$ & $1.00 \mathrm{c}$ & $6.00 \mathrm{j}$ & $3.00 \mathrm{~b}$ & $10.00 \mathrm{c}$ \\
\hline & G-12 & $3.08 \mathrm{c}$ & $1.00 \mathrm{c}$ & $8.25 \mathrm{fg}$ & $3.00 \mathrm{~b}$ & $11.00 \mathrm{~b}$ \\
\hline & G-13 & $1.00 \mathrm{e}$ & $0.00 \mathrm{~d}$ & $9.00 \mathrm{ef}$ & $2.00 \mathrm{c}$ & $9.00 \mathrm{~d}$ \\
\hline & G-14 & $1.00 \mathrm{e}$ & $1.00 \mathrm{c}$ & $11.10 \mathrm{c}$ & $1.00 \mathrm{~d}$ & $8.00 \mathrm{e}$ \\
\hline & G-15 & $4.00 \mathrm{~b}$ & $0.00 \mathrm{~d}$ & $6.00 \mathrm{j}$ & $0.00 \mathrm{e}$ & $6.00 \mathrm{~g}$ \\
\hline & G-16 & $4.00 \mathrm{~b}$ & $1.00 \mathrm{c}$ & $5.00 \mathrm{k}$ & $1.00 \mathrm{~d}$ & $7.00 \mathrm{f}$ \\
\hline & G-17 & $2.00 \mathrm{~d}$ & $0.00 \mathrm{~d}$ & $8.00 \mathrm{gh}$ & $1.00 \mathrm{~d}$ & $8.00 \mathrm{e}$ \\
\hline & G-18 & $3.00 \mathrm{c}$ & $0.00 \mathrm{~d}$ & $7.00 \mathrm{i}$ & $1.00 \mathrm{~d}$ & $6.00 \mathrm{~g}$ \\
\hline & G-19 & $2.00 \mathrm{~d}$ & $0.00 \mathrm{~d}$ & $5.00 \mathrm{k}$ & $3.00 \mathrm{~b}$ & $9.00 \mathrm{~d}$ \\
\hline & G-20 & $2.00 \mathrm{~d}$ & $1.00 \mathrm{c}$ & $6.00 \mathrm{j}$ & $1.00 \mathrm{~d}$ & $10.00 \mathrm{c}$ \\
\hline \multirow{20}{*}{ 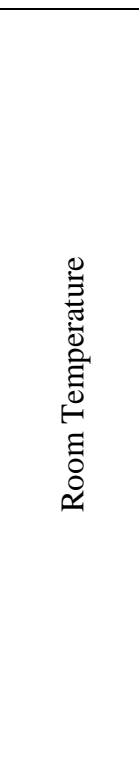 } & G-01 & $5.00 \mathrm{a}$ & $0.00 \mathrm{~d}$ & $9.25 \mathrm{de}$ & $1.00 \mathrm{~d}$ & $8.00 \mathrm{e}$ \\
\hline & G-02 & $4.00 \mathrm{~b}$ & $1.00 \mathrm{c}$ & $8.00 \mathrm{gh}$ & $1.00 \mathrm{~d}$ & $10.00 \mathrm{c}$ \\
\hline & G-03 & $2.00 \mathrm{~d}$ & $1.00 \mathrm{c}$ & $10.00 \mathrm{~d}$ & $3.00 \mathrm{~b}$ & $12.00 \mathrm{a}$ \\
\hline & G-04 & $4.02 \mathrm{~b}$ & $2.00 \mathrm{~b}$ & $12.00 \mathrm{~b}$ & $2.00 \mathrm{c}$ & $11.00 \mathrm{~b}$ \\
\hline & G-05 & $4.00 \mathrm{~b}$ & $3.00 \mathrm{a}$ & $12.00 \mathrm{~b}$ & $3.00 \mathrm{~b}$ & $10.00 \mathrm{c}$ \\
\hline & G-06 & $3.00 \mathrm{c}$ & $1.00 \mathrm{c}$ & $11.00 \mathrm{c}$ & $1.00 \mathrm{~d}$ & $12.00 \mathrm{a}$ \\
\hline & G-07 & $3.00 \mathrm{c}$ & $1.00 \mathrm{c}$ & 9.00 ef & $0.00 \mathrm{e}$ & $11.00 \mathrm{~b}$ \\
\hline & G-08 & $4.00 \mathrm{~b}$ & $0.00 \mathrm{~d}$ & $8.00 \mathrm{gh}$ & $2.00 \mathrm{c}$ & $9.00 \mathrm{~d}$ \\
\hline & G-09 & $5.00 \mathrm{a}$ & $1.00 \mathrm{c}$ & $7.00 \mathrm{i}$ & $1.00 \mathrm{~d}$ & $7.00 \mathrm{f}$ \\
\hline & G-10 & $3.00 \mathrm{c}$ & $0.00 \mathrm{~d}$ & $11.00 \mathrm{c}$ & $2.00 \mathrm{c}$ & $6.00 \mathrm{~g}$ \\
\hline & G-11 & $3.00 \mathrm{c}$ & $0.00 \mathrm{~d}$ & $8.00 \mathrm{gh}$ & $3.00 \mathrm{~b}$ & $9.00 \mathrm{~d}$ \\
\hline & G-12 & $3.00 \mathrm{c}$ & $0.00 \mathrm{~d}$ & 9.00 ef & $3.00 \mathrm{~b}$ & $10.00 \mathrm{c}$ \\
\hline & G-13 & $2.00 \mathrm{~d}$ & $1.00 \mathrm{c}$ & $14.00 \mathrm{a}$ & $1.00 \mathrm{~d}$ & $11.00 \mathrm{~b}$ \\
\hline & G-14 & $2.00 \mathrm{~d}$ & $1.00 \mathrm{c}$ & $12.00 \mathrm{~b}$ & $2.00 \mathrm{c}$ & $12.00 \mathrm{a}$ \\
\hline & G-15 & $2.00 \mathrm{~d}$ & $1.00 \mathrm{c}$ & $7.00 \mathrm{i}$ & $3.00 \mathrm{~b}$ & $8.00 \mathrm{e}$ \\
\hline & G-16 & $5.00 \mathrm{a}$ & $1.00 \mathrm{c}$ & $8.00 \mathrm{gh}$ & $0.00 \mathrm{e}$ & $8.00 \mathrm{e}$ \\
\hline & G-17 & $4.00 \mathrm{~b}$ & $2.00 \mathrm{~b}$ & $10.00 \mathrm{~d}$ & $2.00 \mathrm{c}$ & $9.00 \mathrm{~d}$ \\
\hline & G-18 & $2.00 \mathrm{~d}$ & $1.00 \mathrm{c}$ & $8.00 \mathrm{gh}$ & $1.00 \mathrm{~d}$ & $10.00 \mathrm{c}$ \\
\hline & G-19 & $2.99 \mathrm{c}$ & $2.00 \mathrm{~b}$ & 9.00 ef & $4.00 \mathrm{a}$ & $8.00 \mathrm{e}$ \\
\hline & G-20 & $3.00 \mathrm{c}$ & $1.00 \mathrm{c}$ & $8.00 \mathrm{gh}$ & $2.00 \mathrm{c}$ & $9.00 \mathrm{~d}$ \\
\hline $\mathrm{LSD}_{0.05}$ & & 0.290 & 1.17 & 0.798 & 0.230 & 0.597 \\
\hline $\mathrm{CV}(\%)$ & & 7.64 & 10.14 & 6.98 & 10.31 & 5.09 \\
\hline
\end{tabular}




\section{Conclusions}

From the findings of this study, it was concluded that the storage temperature played an important role in the maintenance of seed health and quality of dhaincha seeds. Fungi of the genera Aspergillus, Fusarium, Rhizopus, and Penicillium were associated with the seed quality of dhaincha seeds. The genotypic difference in seed quality of Sesbania spp. is conspicuous under different storage temperature conditions.

\section{Acknowledgements}

The corresponding author acknowledges greatly the financial support of the Ministry of Science and Technology, Government of the People's Republic of Bangladesh during conduction of this study.

\section{References}

Ahmed, Z. U., Hassan, M. A., Begum, Z. N. T., Khondker, M., Kabir, S. M. H., Ahmad, M. and Ahmed, A. T. A. (eds.). 2009. Encyclopedia of Flora and Fauna of Bangladesh, Vol. 8. Angiosperms: Dicotyledons (Fabaceae-Lythraceae). Asiatic Soc. Bangladesh, Dhaka. pp. 1474.

Alam, M. M., Ali, M. H., Amin, A. K. M. R. and Mirza, H. 2010. Seed quality of green gram stored in different storage environment. Journal of Phytology, 2: 1821.

Baki, A. A. and Anderson, J. D. 1972. Physiological \& biological deterioration of seeds. In Seed Biology, Academic Press, New York. 11: 283-315.

Basavegowda, Gururaj, S. and Arunkumar, H. 2013. Effect of commercial cold storage conditions and packaging materials on seed quality of Chickpea (Cicer arietinum). Global Journal of Science Frontiers Research Agriculture and Veterinary Science, 13: 23-28.
Christensen, C. M. 1967. A note on invasion of durum wheat by storage fungi. Cereal Chemistry, 44: 100-102.

Copeland, L. O. 1976. Principles of Seed Science and Technology. Burgess Pub. Com.

Croft, M., Bickster, A., Manson, J. and Burnette. 2012. Vacuum sealing vs. refrigeration: Which is the most effective way to store seeds? Echo Asia notes. A Regional Supplement to Echo Development notes. 14.

Das, B. K., Barua, I. C. and Dey, S. C. 1998. Effect of packing material, storage condition and duration of storage on seed viability, vigour and seedling survivability in Rajmah (Phaseolus vulgaris). Legume Research, 21: 91-95.

Dwivedi, S. N. and Shukla, T. N. 1990. Effect of methods of storage on germinability and mycoflora of gram (Cicer arietinum). Seed Research, 18: 82-85.

ISTA. 2001. International Rules of Seed Testing Association. Procedure of International Seed Testing Association. 24-48.

Khalequzzaman, K. M. 2004. Biological control of foot and root rot of French bean by using antagonist (Rhizobia). Ph.D. disser., Dep. Plant Path., Bangladesh Agril. Univ., Mymensingh, Bangladesh.

McDonald, M. B. 2004. Orthodox seed deterioration and its repair. In: BenechArnold, R. L. and Sanchez, R. J. (eds.). Handbook of Seed Physiology Applications to Agriculture. Food Products Press and Haworth Reference Press, New York. pp. 273-296.

Monira, M. H., Amin, A., Aktar, M. M. and Mamun, M. A. A. 2012. Effect of containers on seed quality of storage soybean seed. Bangladesh Research Publication Journal, 7: 421-427. 
Effect of storage condition on dhaincha seed health and quality

Sarwar, A. K. M. Golam, Islam, A. and Jahan, S. 2015. Characterization of dhaincha accessions based on morphological descriptors and biomass production. Journal of the Bangladesh Agricultural University, 13: 55-60.

Sharma, S. N., Goyal, K. C., Gupta, I. J., Karkralya, B. L., Sharma, S. K., Mehta, S. M. and Rathore, A. K. S. 1998. Packaging material and soybean seed quality during storage. Seed Research, 26: 89-91.
Karim, M. T. 2015. Effect storage condition on seed physiological and pathological characteristics of some Dhaincha germplasms. MS Thesis, Dep. Seed Sci. Tech., Bangladesh Agril. Univ., Mymensingh, Bangladesh.

Veasey, E. A., Schammass, E. A., Vencovsky, R., Martins, P. S. and Bandel, G. 1999. Morphological and agronomic characterization and estimates of genetic parameters of Sesbania Scop. (Leguminosae) accessions. Genetics and Molecular Biology, 22: 81-93. 